\title{
INTERPOSITION OF THE COLON BETWEEN LIVER AND DIAPHRAGM (CHILAIDITI'S SYNDROME) IN CHILDREN
}

\author{
BY \\ A. D. M. JACKSON and C. J. HODSON \\ From the Institute of Child Health, University of London, and the Queen Elizabeth Hospital for Children, \\ London
}

(RECEIVED FOR PUBLICATION SEPTEMBER 24, 1956)

In 1910 Chilaiditi published a paper in which he described three adult patients with gaseous abdominal distension and intermittent displacement of the liver by a distended loop of colon. Although there had been some earlier brief reports, for example those of Gontermann (1890), Cohn (1907) and Weinberger (1908), this was the first really detailed account of the condition which has come to be known as Chilaiditi's syndrome.

During the last 45 years many more cases have been reported but very few of these have been children. It will be of some interest, therefore, to record the clinical and radiological findings in four further cases of Chilaiditi's syndrome in childhood and to discuss the aetiology of the condition as suggested by the study of these children.

\section{Case Reports}

Case 1. Andrew F. was born in August, 1949. He was first seen in December, 1950, at the age of 16 months. Abdominal distension had been noticed for about a year and more recently there had been some abdominal pain and vomiting. The past history was otherwise uneventful and the parents and a sibling were healthy.

On examination the abdomen was considerably distended and tympanitic. With the child lying supine the liver edge could be felt below the right costal margin. In the erect and left lateral positions, however, there was resonance to percussion over the right hypochondrium continuous with the resonance over the lung, and the edge of the liver could be palpated with some difficulty on the left side of the abdomen. The urine was normal and there were no other important findings.

RADIOLOGICAL ExAMINATION. Radiological investigations were carried out on several occasions over a period of several months. There was invariably gaseous distension of the stomach and intestines. Films taken in the erect position showed the liver to be displaced downwards and to the left, its place being taken by a distended loop of colon (Fig. 1). In the supine position the liver and colon usually occupied their normal positions. On one occasion during the fluoroscopy the ascent of the colon and the displacement of the liver were actually observed as the patient was rotated from the supine to the left lateral position. Fluoroscopy after an opaque meal excluded any form of intestinal obstruction and the interposed loop of bowel was identified as the hepatic flexure.

Progress. During the six months following his first visit to hospital the child had recurrent attacks of severe abdominal pain with vomiting and his mother became aware of his swallowing air. He was admitted to hospital in June, 1951, and put to bed. The attacks promptly ceased, although the abdominal distension persisted and the position of the liver could still be altered with posture.

The patient's progress over the next few years was satisfactory and when last seen in April, 1956, he was having only occasional, mild abdominal pain in the evening and this was rapidly relieved by sitting or lying

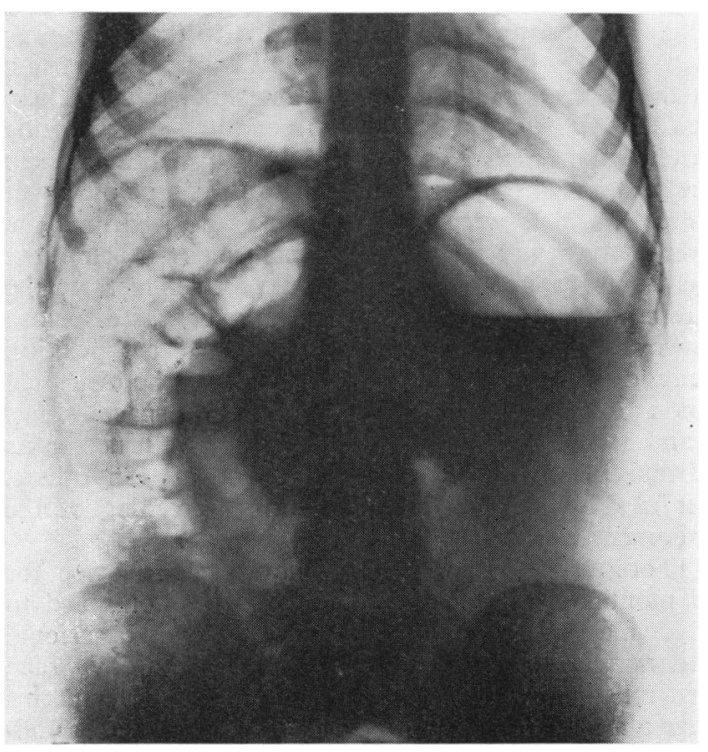

Fig. 1.-Radiograph of the abdomen (erect) in Case 1. 
down. His mental and physical development was normal. However, apart from considerable diminution of the abdominal distension, the clinical and radiological findings were unchanged.

Case 2. Ann S. was born in November, 1951. She was admitted to hospital in November, 1954, at the age of 3 years with a history of abdominal distension for most of her life and recent abdominal pain. The mother had also noticed that the child gulped her food and frequently passed flatus, especially if any pressure was applied to the abdomen. The bowels were constipated but there was no history of vomiting. Details of the family and past history were not relevant.

On examination the abdomen was extremely distended. A few dilated veins were seen on the upper abdominal wall and there was visible intestinal peristalsis. The liver was displaced downwards and to the left in the erect position, its lower edge being palpable in the left hypochondrium. Occasionally with the patient lying down the liver could be felt in the normal position. On percussion the whole abdomen was tympanitic and there was no liver dullness. During her stay in hospital the child occasionally complained of slight abdominal pain in the evening. Relief of the constipation did not affect the abdominal distension which was found to be much less marked in the early morning and to increase gradually during the day irrespective of whether the child was kept in bed or allowed up. The displacement of the liver, however, was only demonstrated in the erect position.

Radiological Examination. In view of the experience with Case 1 radiological investigations were more extensive in this case. Comparison of films taken soon after the child awoke in the early morning with those taken in the late afternoon showed a striking difference in the degree of the abdominal distension. In the morning there was very little gas in the stomach and only slight distension of the colon confined to the descending part, whereas later in the day there was gross distension of the stomach and most of the intestinal tract. These findings confirmed the clinical observation of increasing distension during the day. There were also differences in the position of the liver depending on the time of day at which the films were taken. In the early morning the liver shadow was seen in its normal position below the right diaphragm in both supine and erect films. In the afternoon with the patient erect the liver was displaced downwards, forwards and medially, its exact position being difficult to make out, and a loop of the distended colon occupied the sub-diaphragmatic space (Figs. 2 and 3); in the supine position the liver returned to its normal place on some occasions but not on others. Air swallowing was observed during screening of the atdomen.

Progress. This patient has now been followed for 18 months. For the first year or so she continued to have marked abdominal distension, and occasional abdominal pain during the later part of the day which was relieved by lying down. Recently the symptoms have almost completely subsided but at the latest examination in April, 1956, although the abdominal distension was considerably less, the clinical and radiological signs of interposition with the liver displacement were unaltered.

Case 3. Naomi P. was born in December, 1950, and was 5 years old when first admitted to hospital. Her complaint was of attacks of abdominal pain which had begun some five weeks previously. The pain was quite severe, lasted about half an hour and was relieved by belching and passing flatus; it occurred most commonly in the late afternoon. She never vomited but was sometimes constipated and her appetite, previously good, had become poor. Her abdomen had been unduly distended for as long as the parents could remember. There were no siblings and the family and past history were not relevant to the present condition.

The clinical findings were similar to those in the previous cases. The increasing daytime distension of the abdomen was particularly well marked, the circumference of the abdomen increasing from 18 in. at 5 a.m. to 24 in. at 2 p.m. In the erect position and occasionally in the afternoon in the supine position the liver dullness was absent and the liver itself was not palpable. The child's physical and mental development was normal. There were no abnormal physical signs and laboratory examination of the urine and faeces showed no abnormality.

Radiological Examination. The typical features of the syndrome were again demonstrated. There was progressive daytime distension of the whole alimentary tract. Supine films (Fig. 4) showed the normal anatomical relations of liver and colon but in the erect position the colon was interposed between liver and diaphragm with displacement of the liver (Fig. 5). On one occasion the interposition was only partial, the liver being displaced forwards but not downwards. An opaque meal passed down the alimentary tract without obstruction and air swallowing was observed during the examination.

Progress. This child's progress has so far been followed for only three months. During this time some attempt has been made to lessen the abdominal distension without much success. Oral administration of charcoal biscuits and a carminative mixture produced no obvious improvement, and the wearing of an elastic abdominal belt has, if anything, increased the child's discomfort. She still vomits occasionally and is troubled by the progressive daytime distension but is otherwise quite well and has gained weight.

Case 4. Elizabeth F. was born in September, 1951, and was $4 \frac{1}{2}$ years old when admitted to hospital in February, 1956. Following an attack of gastro-enteritis two months before admission she had complained of anorexia, recurrent abdominal pain and vomiting, frequent passage of flatus and abdominal distension, which was most marked at the end of the day. For some time past she had been noticed to swallow air. The parents and a sibling were healthy and there were no incidents of note in the past history.

On examination the physical signs of liver displacement were present in the erect position and the typical progressive abdominal distension was observed. The 


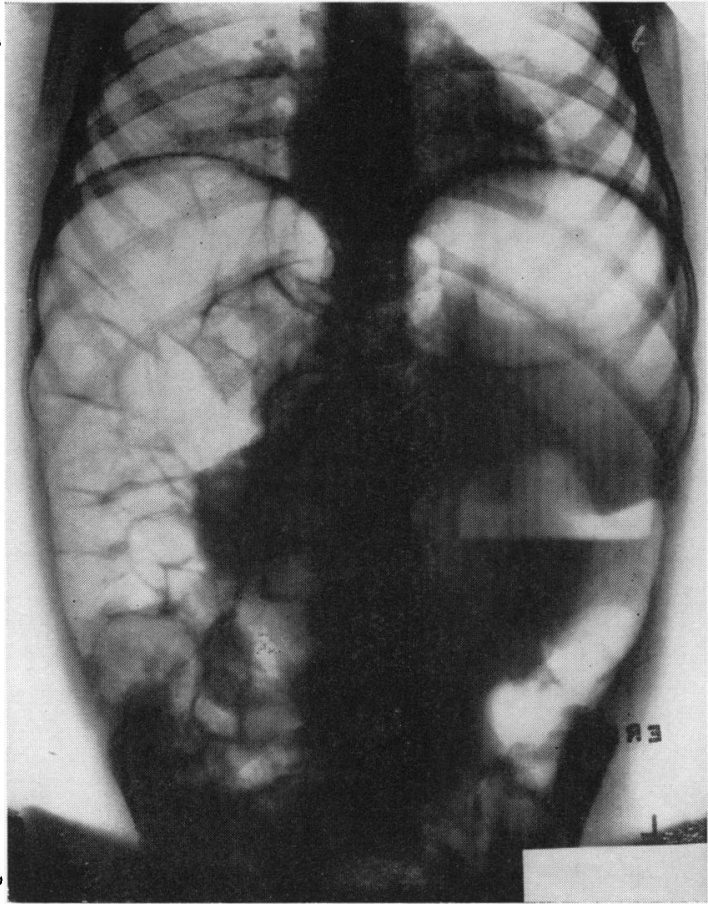

FIG. 2.

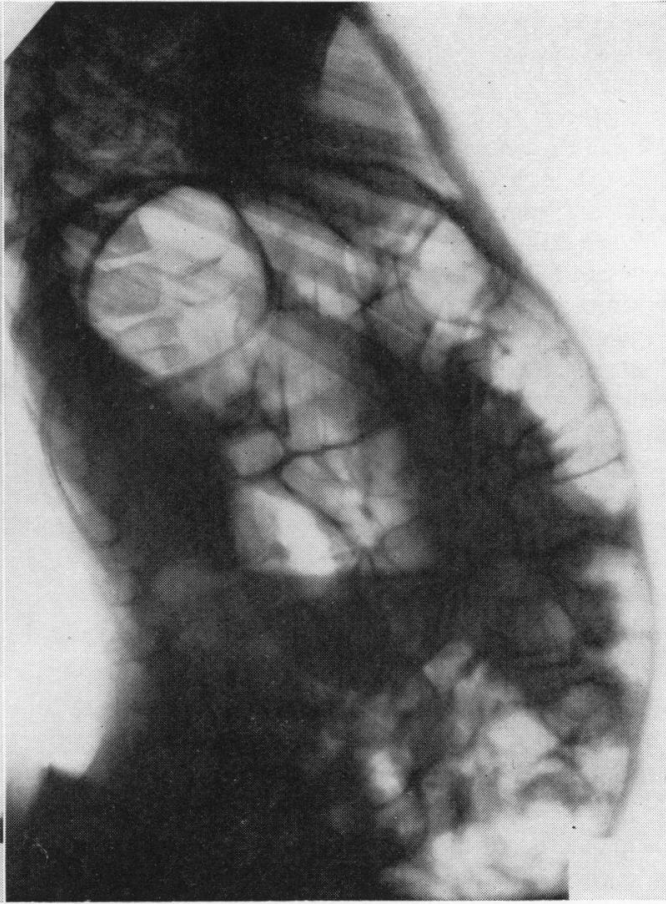

FIG. 3 .

Figs. 2 and 3.-Antero-posterior and lateral radiographs of the abdomen (erect) in Case 2. These films were taken in the late afternoon and illustrate the extreme degree of gaseous distension of the stomach and intestines.

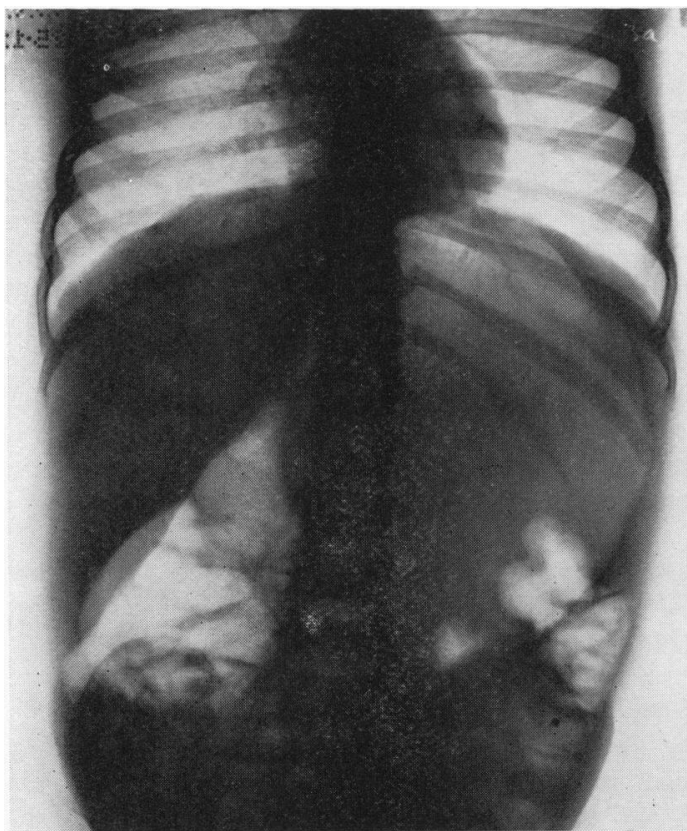

FIG. 4.

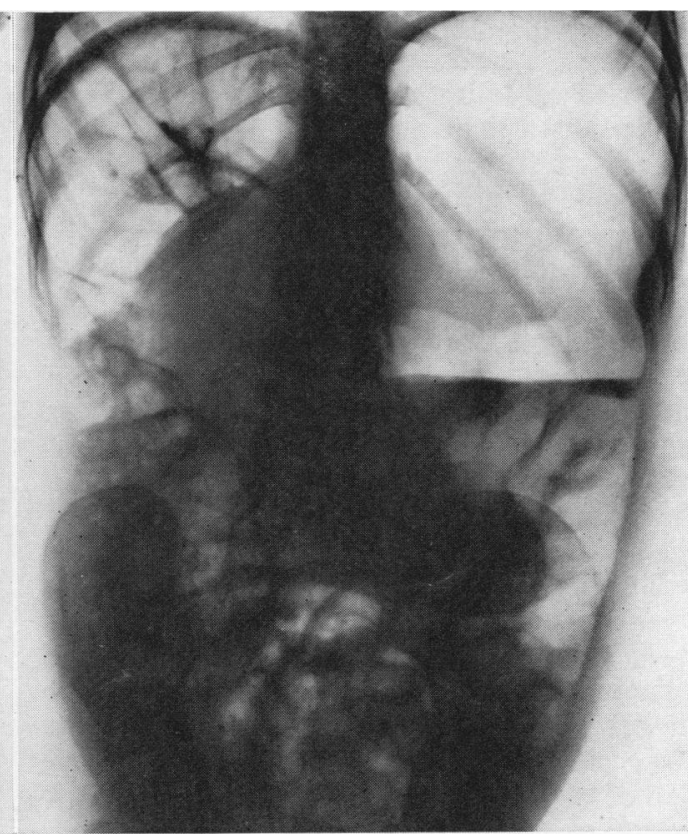

FIG. 5.

Figs. 4 and 5.-Radiographs of the abdomen (supine and erect) in Case 3 . In the supine position (Fig. 4 ) the liver and colon occupy their normal positions. In the erect position (Fig. 5) there is interposition of the colon and displacement of the liver. The huge gas bubble in the stomach is typical of aerophagy. 
urine was normal and there were no other abnormalities on clinical examination.

RAdiological Examination. The familiar radiological features were present (Fig. 6). Gaseous distension of the alimentary tract was far greater in the later part of the day. Air swallowing was observed on screening and there was no evidence of intestinal obstruction. Interposition of the colon and varying degrees of displacement of the liver was seen in films taken with the patient erect; the normal anatomy was restored in the supine position.

Progress. This patient has been under observation for about four months. During this time she has made a complete recovery. She wore an abdominal belt for three months after her discharge from hospital but had left it off for one month before the last examination when she was found to have no clinical or radiological abnormality.

\section{Discussion}

These cases illustrate a well-defined clinical syndrome, the main features of which are marked diurnal abdominal distension and interposition of the colon between the liver and the diaphragm in the erect position. The commonest symptom is abdominal pain which is sometimes quite severe and associated with vomiting. This pain occurs typically towards the end of the day when the abdominal distension is maximal and is less frequent and much less severe when the patient is in bed. Other, less common symptoms are anorexia, constipation and the frequent passage of flatus. The distinctive physical signs are those of abdominal distension with absent liver dullness, and the liver edge may be palpable somewhere on the left side of the abdomen. There are no other abnormalities or signs of retardation of physical or mental development.

The diagnosis may be confirmed by radiology if, in the erect position, the striking appearance of a distended loop of colon under the right dome of the diaphragm is present and the liver is to a greater or lesser degree displaced. In addition, haustral markings establish the fact that the subphrenic gas is within the colon and not free in the peritoneal cavity. The generalized nature of the distension, which extends right down to the lower rectum, and the absence of large faecal masses help to distinguish the radiological picture from that of Hirschsprung's disease and chronic constipation of the 'terminal reservoir' type. It is important to ensure that radiographs are taken in the erect position and that a lateral view is included, since interposition does not usually occur when the patient is supine and the position of the liver may be in doubt on an anteroposterior film.

The literature on interposition of the colon in adults is extensive and several authors have each collected over 20 cases (Uspensky, 1928; Trémolières and Tardieu, 1931; Kolju, 1938; Hubacher, 1946; Torgersen, 1951). The incidence of the condition in adults appears to be about 1 per 1,000 radiological examinations made in the erect position, i.e., chest films or screening (Uspensky, 1928; Kolju, 1938). This figure might, however, be considerably less if lateral radiographs had more often been taken to confirm the displacement of the liver. A distended colon lying anterior to the liver, and therefore not truly interposed, may in an antero-posterior film obscure the liver shadow, giving the false impression that the liver is displaced. The scarcity of published reports concerning children suggests that the condition is rare in this age group but cases may have been missed because radiographs of children with abdominal distension are not normally taken in the erect position. The fact that the present series of four cases* was collected over the space of only a few years suggests that such cases are not exceptionally uncommon in children. In adults both sexes are affected, males more frequently than females, and there is a suggestion of a geographical distribution in the fact that cases have been reported most frequently by authors on the continent of Europe. A recent extensive review of the subject (Baum and Karpati, 1954) lists 57 references of which only four are in the English language.

Baum and Karpati (1954) discuss the aetiology of Chilaiditi's syndrome in some detail. They point out that the liver is held in position mainly by a sort of sub-diaphragmatic suction and suggest that certain abnormalities can disturb this relationship between liver and diaphragm, allowing the liver to descend under the influence of gravity and the colon to take its place. Such abnormalities, which may act singly or in combination, include intestinal distension, diaphragmatic paralysis, abnormalities of the size or suspension of the liver, traction on the liver by adhesions in the region of the duodenum, and ascites. The evidence that some of these factors, e.g., abnormal suspension of the liver and the traction effect of the peri-duodenal adhesions, play any part is, however, unconvincing.

There may be different degrees of interposition and liver displacement. In the complete form, illustrated by the present cases, the colon lies above the liver, contact between liver and diaphragm is broken and the liver is displaced downwards, forwards and medially. Interposition is incomplete or partial when the colon does not get above the liver but lies laterally (Fig. 7) or posteriorly (Figs. 8 and 9). The liver is then displaced medially or

* In addition to the four cases studied in detail three further cases of complete interposition have come to our notice during the preparation of this paper and one case of partial interposition is briefly described in the legend to Fig. 7. 


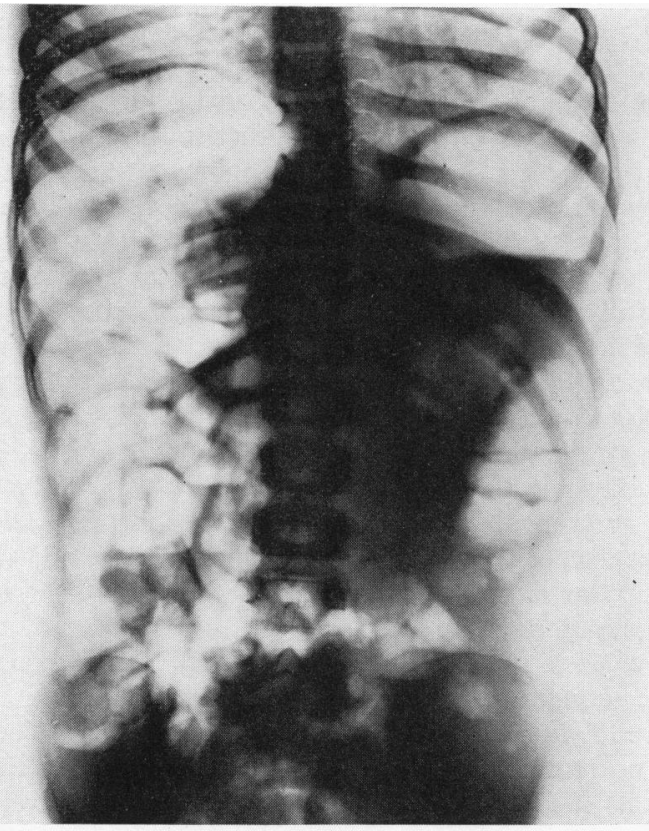

FIG. 6.

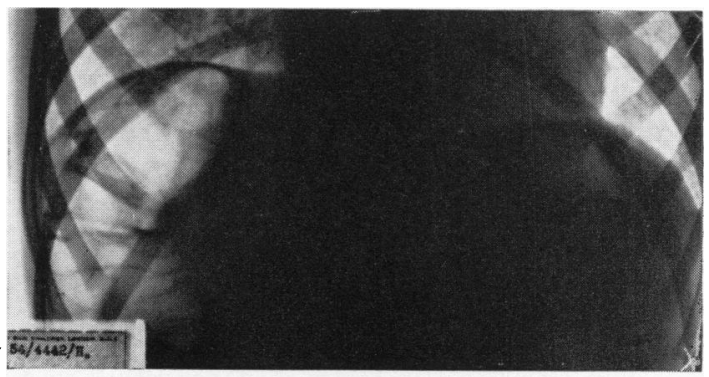

FIG. 7.

FIG. 6.-Radiograph of the abdomen (erect) in Case 4

Fig. 7.-Part of a radiograph of the abdomen (erect) of a girl aged 8 years showing a partial (lateral) interposition of the colon with medial displacement of the liver. This was a chance finding; the patient had pneumonia and there were no abdominal symptoms. The interposition has not been seen again despite repeated radiological examinations.

Figs. 8 and 9.-Antero-posterior and lateral radiographs of the abdomen (erect) in Case 3. On this occasion the interposition was incomplete (posterior). The colon has risen behind but not above the liver which is displaced forwards.

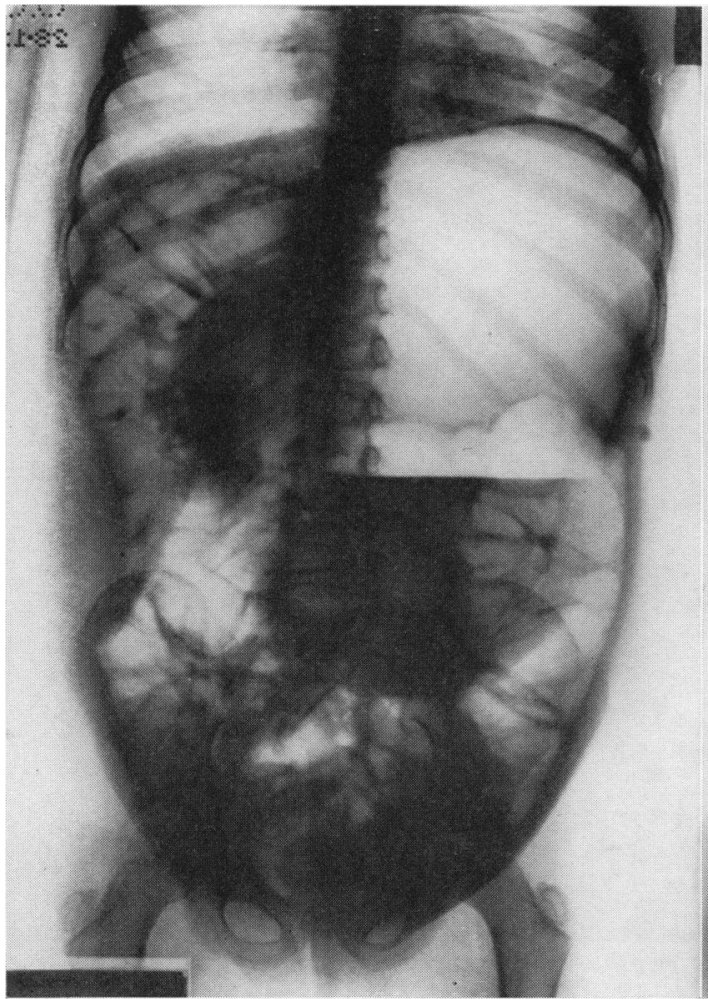

FIG. 8 .

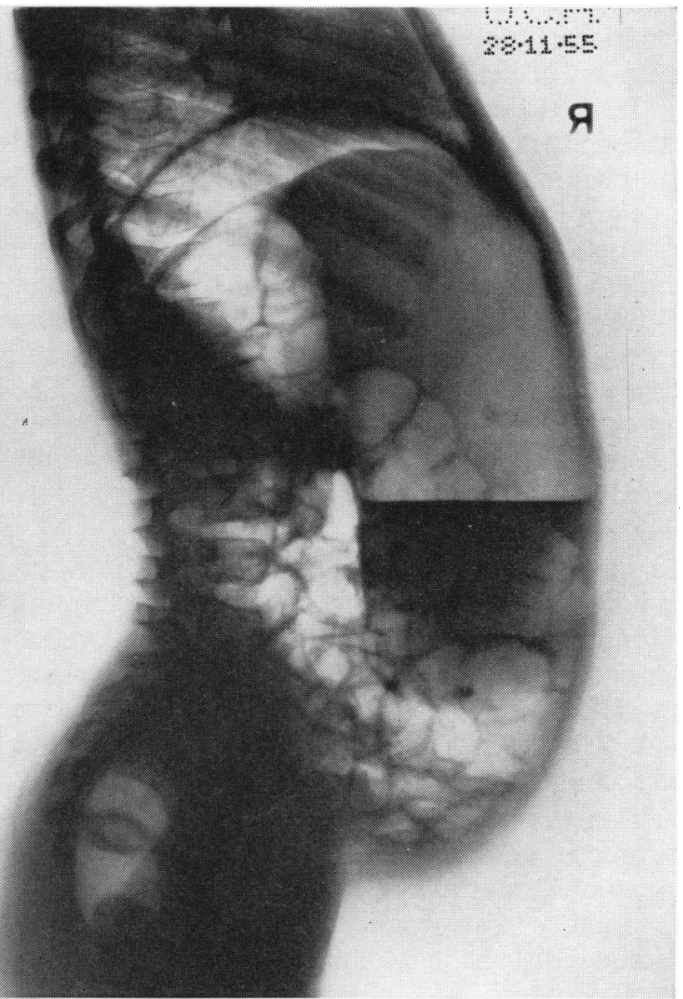

FIG. 9. 
forwards but not downwards. Chilaiditi's (1910) original cases were of this type. Presumably such factors as the intra-abdominal pressure, the degree of distension of the stomach and intestines and the mobility of the colon determine whether the interposition shall be partial or complete. Occasionally the interposition is transient, being detected for only brief periods without further recurrence. In such cases there is usually a very temporary cause for the distension of the gut such as an acute respiratory infection (Grüber, 1937; see also Fig. 7) or pregnancy (Hunter and Rapp, 1953). In most cases, however, including the four children under discussion, the interposition persists, although only in the erect position, over a much longer period and occasionally the colon becomes permanently fixed in its abnormal position by adhesions between it and the liver or diaphragm (Rogers, 1935; Choussat and Choussat-Clausse, 1937). Very rarely other parts of the intestinal tract, such as the ileum (Baum and Grasser, 1952), may become interposed.

Abdominal symptoms such as pain, vomiting, flatulence and constipation, were present in about half the reported adult cases and in addition to the development of adhesions a further important complication may arise, namely torsion of the interposed loop of bowel which gives rise to the signs and symptoms of acute intestinal obstruction. This may lead to an unnecessary laparotomy either because in the radiograph the presence of a gas-filled loop of colon under the diaphragm is misinterpreted as free gas in the peritoneal cavity (Pendergrass and Kirk, 1929) or because the importance of posture in the relief of interposition is not realized. Hunter and Rapp (1953) reported a case of temporary interposition with torsion of the interposed hepatic flexure demonstrated by barium enema. The symptoms of obstruction were rapidly relieved when the colon was released and the normal anatomy restored by alteration of the patient's posture during radiological examination.

As far as children are concerned very little has been added in recent years to the clinical description by Gontermann (1890) of an infant with abdominal distension and the physical signs of liver displacement (Wanderleber). Grüber (1937) and Arce (1946) also described cases and in addition gave brief accounts of the radiological findings.

Such is the historical background of Chilaiditi's syndrome in adults and children against which the observations on the present cases were analysed. The primary abnormality in these children is abdominal distension almost certainly due to the aerophagy which was observed in all four cases either by the parents or by the radiologist. The progressive distension of the alimentary tract during the day with tremendous dilatation of the stomach, the diminution of the distension by night and the absence of intestinal obstruction are further facts in support of a diagnosis of aerophagy. The reason why these children should acquire the air swallowing habit to such a marked degree is not obvious. None of them appeared to have any significant emotional disturbance to suggest a psychological cause. Since the abdominal distension persists even when the patient is kept in bed it is apparently independent of colonic interposition and liver displacement which only take place in the erect position. Kolju (1938) studied the mechanism of interposition in adults by fluoroscopy. With the patient upright the colon was distended with air through a rectal tube and was seen to rise up and insert itself between the liver and the diaphragm. At this point displacement of the liver took place. It is probable that this is exactly the sequence of events in the present cases with air swallowing as the cause of the distension. There is no reason to suggest any abnormality of the liver or its suspensory ligaments since the normal liver is sufficiently mobile to allow considerable displacement to take place as, for example, in cases of pneumoperitoneum either artificially induced (Fig. 10) or spontaneous (O'Donoghue, 1956).

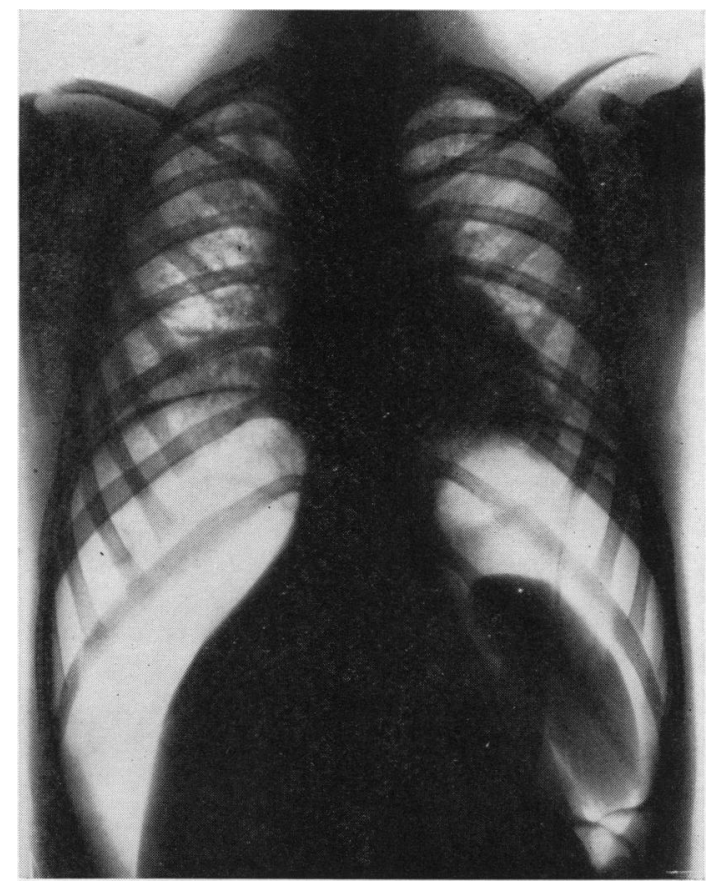

FIG. 10.-Radiograph of the chest and abdomen of a boy aged 12 in whom an artificial pneumoperitoneum has been induced for pulmonary tuberculosis. There is considerable infero-medial displacement of the liver. 
One further fact requires explanation. In a search for further examples of this syndrome radiographs, taken in the erect position, of other children with abdominal distension were examined. These included 10 cases of Hirschsprung's disease and four cases of aerophagy, in all of which the distension of the colon was comparable with that of the cases of Chilaiditi's syndrome. Nevertheless, in no instance was there any evidence of interposition or liver displacement. In addition, therefore, to colonic distension and the effect of gravity some further factor is necessary if interposition is to take place. It seems probable that this factor is an unusual mobility of the ascending colon and hepatic flexure due to the presence of an ascending mesocolon. There is evidence from operation reports (Rogers, 1935; Herget, 1951) and necropsy reports (Pendergrass and Kirk, 1929; Soupault, 1930) that this mobility of the colon exists in adult cases. In children a highly-placed caecum and an apparent redundancy of the hepatic flexure are normal anatomical variants (Bryant, 1924; Caffey, 1956) so that abdominal distension might by chance occur in a child with a mobile ascending colon.

It is concluded, therefore, that in the present group of children the aetiology of interposition of the colon is primarily abdominal distension of a severe degree resulting from aerophagy. Unusual mobility of the ascending colon combined with the effect of gravity result in ascent of the distended bowel into the sub-diaphragmatic space with consequent infero-medial displacement of the liver. These abnormalities associated with the symptoms and distinctive radiological picture already described constitute a particular form in children of the syndrome which is probably best termed 'interposition of the colon between liver and diaphragm' or more simply 'interposition of the colon'. This title is preferable on the grounds of euphony to 'hepatodiaphragmatic interposition of the colon' which has been used by some authors. The eponymous title 'Chilaiditi's syndrome' is not strictly correct since the cases described by Chilaiditi (1910) were adults with a partial form of interposition and not entirely comparable with those under discussion.

The pain and vomiting in these children could be entirely explained by the degree of intestinal distension and it might be argued that the abnormal position of the liver and colon play no part in their production. However, children with quite marked abdominal distension due to aerophagy without interposition seldom complain of severe pain, whereas in three of the present cases (Cases 1, 3 and 4) the symptoms were at times bad enough to suggest the possibility of intestinal obstruction. In Case 1, before the nature of the diagnosis has been appreciated, laparotomy was seriously considered and was only avoided by the timely disappearance of the symptoms after the patient had been put to bed. The frequent observation of this relief of acute abdominal symptoms by a change of posture from upright to supine indicates that, at least on some occasions, the symptoms are due to colonic or liver displacement rather than to distension. Since experience in patients with artificial pneumoperitoneum suggests that liver displacement per se does not cause abdominal pain it is probably the interposition of the colon which is to blame. A possible explanation is that the symptoms are due, as in certain adult cases previously mentioned, to obstruction brought about by torsion or incarceration of the interposed loop of colon as it becomes more and more distended in the relatively confined space above the liver. Releasing the interposition by placing the patient supine would relieve this obstruction and hence the symptoms. The amount of distension varies from case to case and from time to time in any one case and it is possible to imagine a degree of distension of the colon sufficient to give rise to interposition but not severe enough to cause obstruction. Hence the fact that in Cases 1 and 2 there has been a reduction in distension and in the severity and frequency of symptoms, although in both cases interposition of the colon persists. This theory would also account for the absence of symptoms in many adult cases and for such cases as the child whose radiograph is illustrated in Fig. 7, in which the distension is minimal and the resulting interposition incomplete and symptomless.

In view of the relatively short period of observation and the small number of children studied it is not possible to be certain of the natural history of the syndrome in these children. The anatomical abnormality has so far persisted for five and a half years in Case 1 and for 18 months in Case 2, although in both cases the air swallowing and consequently the distension and pain are steadily diminishing. If this improvement is maintained and the colon becomes less mobile a spontaneous cure, such as appears to have occurred in Case 4 , might be expected. If, however, the mobility of the colon is permanent the persistence or recurrence of interposition would always be possible depending on the presence of abdominal distension, whether it were due to aerophagy or to any other cause. Since the condition is comparatively common in adults it is possible that some childhood cases do, in fact, persist into adult life.

Treatment for the condition has been attempted 
in only two of the children (Cases 3 and 4). In the light of the spontaneous improvement in Cases 1 and 2 the necessity for any treatment is doubtful and the assessment of its value difficult. Prevention of the aerophagy is obviously desirable but in children of this age difficult to achieve. Carminatives had no effect on the intestinal distension in Case 3. The effect of wearing an abdominal belt was tried in Cases 3 and 4, and, although in Case 4 this measure was accompanied by an apparent recovery, it is difficult to believe that this was anything but coincidental. On balance there does not so far appear to be any significant difference in progress between the treated and untreated cases. Operative fixation of the colon (Rogers, 1935) or liver (Schenck, 1938) has been suggested to prevent interposition taking place and to avoid complications. Until the possibility of permanent spontaneous cure is excluded such drastic therapy does not seem to be justified. Surgical measures to divide established adhesions between the colon and the diaphragm would perhaps be more reasonable but it is difficult to see how this complication would be diagnosed on clinical grounds.

In conclusion it is suggested that the syndrome of interposition of the colon in children is a definite entity, easily recognized by a typical clinical and radiological picture, and is of some importance by reason of the symptoms it may produce and the possible complication of intestinal obstruction which may lead to an unnecessary laparotomy if the nature of the condition is unrecognized. It is very probable that more cases would be discovered if the existence of the syndrome were more widely appreciated.

\section{Summary}

The syndrome of interposition of the colon between liver and diaphragm is described.
A clinical and radiological study of four cases of this syndrome in childhood is reported. It is concluded that in these cases the primary abnormality is abdominal distension due to aerophagy and that unusual mobility of the distended colon permits its ascent into the sub-diaphragmatic space in the erect position, with consequent displacement of the liver.

The syndrome is probably not uncommon in children despite the rarity of published reports.

We are indebted to the following paediatricians who have allowed us to study and record their cases and have given much helpful advice in the preparation of this paper: Dr. Helen Mackay (Case 1), Dr. Richard Dobbs (Case 2), Dr. Bernard Schlesinger (Case 3), Dr. George Ormiston and Dr. Wilfrid Sheldon (Case 4). We should also like to thank Drs. W. F. Richards and B. C. H. Ward of High Wood Hospital for Children for their advice concerning artificial pneumoperitoneum and for the radiograph reproduced in Fig. 10. Dr. L. G. Blair kindly provided several of the other radiographs.

\section{REFERENCES}

Arce, G. (1946). Acta pediatr. esp., 4, 747.

Baum, G. and Grasser, H. (1952)., Fortschr. Röntgenstr., 77, 616. and Karpati, A. (1954). Med. Mschr., 8, 221

Bryant, J. (1924). Amer. J. med. Sci., 167, 499.

Caffey, J. (1956). Pediatric $X$-ray Diagnosis, 3rd ed., p. 546. Chicago.

Chilaiditi, D. (1910). Fortschr. Röntgenstr., 16, 173.

Choussat and Choussat-Clausse (1937). Bull. Soc. Radiol. méd. Fr., 25, 147.

Cohn, M., (1907). Verh. dtsch. RöntgGes., 3, 66

Gontermann (1890). Dtsch. med. Wschr., 16, 1043.

Grüber, E. (1937). Ibid., 63, 1113 .

Herget, R. (1951). Beitr. klin. Chir., 183, 83.

Hubacher O. (1946). Schweiz, med. Wschr. 76, 554

Hunter, H. L. and Rapp, R. (1953). Radiology, 61, 67.

Kolju, K. J. (1938). Amer. J. Roentgenol., 39, 928.

O'Donoghue, P. D. (1956). Lancet, 1, 189.

Pendergrass, E. P. and Kirk, E. (1929). Amer. J. Roentgenol., 22,238 .

Rogers, J. C. T. (1935). Illinois med. J., 68, 264.

Schenck, S. G. (1938). Arch. Surg. (Chicago), 36, 766

Schenck, S. G. (1930). Arch. Mal. Appar. dig., 20, 350

Soupault, R. (1930). Arch. Mal. Appar. dig., 20, 350.

Trémolières, F. and Tardieu, A. (1931).' Arch. Mal. Appar. dig., 21, 1154

Uspensky, A. (1928). Fortschr. Röntgenstr., 37, 540

Weinberger, M. (1908). Med. Klin., 4, 584. 